\title{
Evaluación del aprendizaje del tema componentes invariantes del dato científico en la asignatura metodología de la investigación
}

\author{
Cerdán $\mathrm{MS}^{1}$, Di Masso $\mathrm{RJ}^{1}$, Lapalma MA ${ }^{1 *}$ \\ ${ }^{1}$ Cátedra de Metodología de la Investigación. Facultad de Ciencias Veterinarias, Universidad Nacional \\ de Rosario (FCV-UNR), Casilda, Argentina. \\ * Correspondencia: María Alejandra Lapalma. FCV-UNR, Ovidio Lagos y Ruta 33, 2170 Casilda, Santa \\ Fe, Argentina. E-mail: alelapalma@hotmail.com
}

Recibido: 1 Junio 2020. Aceptado: 30 de Octubre 2020. Disponible en línea: 9 Marzo 2021 Editor: R. Sobrero

RESUMEN. Se evaluó el aprendizaje del tema componentes invariantes del dato científico en el examen final de estudiantes que rindieron la asignatura Metodología de la investigación en la carrera de Medicina Veterinaria de la Universidad Nacional de Rosario. Se utilizó un instrumento especialmente diseñado, conformado por un enunciado consistente en un texto que incluye los elementos vinculados con cada uno de los componentes invariantes del dato científico (entidad, propiedad, estado y procedimiento), una consigna de trabajo y una tabla para completar por parte del estudiante como respuesta. De los 57 estudiantes evaluados el $38,6 \%$ respondió correctamente. El $26,3 \%$ reconoció la entidad y de ellos el $13,3 \%$ también reconoció la propiedad y su valor, pero no el procedimiento y el $86,7 \%$ identificó el procedimiento y el estado, pero no la propiedad. En el $35,1 \%$ de los casos correspondientes a estudiantes que no identificaron correctamente la entidad, el 35,0 \% identificó la propiedad y el 65,0\%, sin reconocer ni la entidad ni la propiedad, indicó correctamente el estado y el procedimiento. Estas inconsistencias ponen de manifiesto la atomización del proceso de razonamiento frente al desafío del problema a abordar, a la vez que evidencian un conocimiento frágil.

SUMMARY. Assessment of the learning of the topic 'invariant components of scientific data' in research methodology teaching. Learning evaluation of the topic "invariant components of scientific datum" in a Research methodology course. The learning of the topic invariant components of scientific datum was evaluated in the final exam of students who regularized the subject Methodology of research in the career of Veterinary Medicine of the National University of Rosario. A specially designed instrument was used, consisting of a text that includes the elements linked to each of the invariant components (entity, property, state and procedure), a work slogan and a table to complete by student's responses. Of the 57 students evaluated, $38.6 \%$ responded correctly. $26.3 \%$ recognized the entity and $13.3 \%$ of them also recognized the property and its value, but not the procedure and $86.7 \%$ identified the procedure and the state, but not the property. In $35.1 \%$ of the students who did not identify correctly the entity, $35.0 \%$ identified the property and $65.0 \%$, without recognizing either the entity or the property, correctly indicated the status and the procedure. These inconsistencies show the atomization of the reasoning process in face of the challenge of the problem to be addressed, and at the same time evince the existence of fragile knowledge.

Palabras clave: pensar acerca de la ciencia, conocimiento olvidado, conocimiento ritual

Keywords: thinking about science, forgotten knowledge, ritual knowledge

\section{Introducción}

La enseñanza de la metodología de la investigación científica en el nivel universitario puede enfocarse al menos desde dos puntos de vista: como un requerimiento vinculado con el "hacer ciencia" o como un conjunto de saberes que den fundamento a "pensar acerca de la ciencia". La primera opción suele estar asociada a aquellos estudiantes que, durante su formación de grado, incursionan en tareas de producción de conocimiento crítico $y$, por ende, está restringida a un conjunto limitado de participantes. La segunda, por el contrario, atraviesa todo el currículo universitario en tanto enfrenta al estudiante con el modo de producción del tipo particular de conoci- miento con el que se vincula a lo largo de su formación como futuro profesional. La toma de conciencia acerca de cómo se genera la información que recibe de sus docentes o a la que accede en los textos, lo debería alertar respecto de su provisionalidad y obsolescencia, y sobre la necesidad ineludible de la actualización permanente. En este contexto, la expresión toma de conciencia no remite necesariamente "al mecanismo piagetiano de toma de conciencia" (Taborda Charrúa, 2010) sino que se utiliza con un sentido pragmático, como expresión de uso habitual en el lenguaje cotidiano, para indicar la acción de darse cuenta de la importancia de algo. La referencia a la actualización permanente suele ser utilizada en las aulas universitarias como una muletilla no siempre fundamentada. 
En este sentido, pensar colaborativamente acerca de la ciencia puede ser una estrategia tendiente a construir dicho fundamento.

Un rasgo distintivo que, como tal, caracteriza a la actividad científica en el campo de las ciencias fácticas, es la contrastación empírica. Brevemente, la misma consiste en someter las presunciones o conjeturas enunciadas como hipótesis, al dictamen de los hechos. Si bien las técnicas de contrastación empírica son propias de cada ciencia y, dentro de ellas, de cada disciplina, en todos los casos sirven para enfrentar ciertas ideas acerca del comportamiento de algún aspecto de la realidad con determinados hechos mediante la experiencia. Cualquiera sea la técnica utilizada en cada caso particular que se analice, la misma permite al investigador disponer de datos. Todo dato es una unidad de información y, por más simple que parezca, los datos, pese al origen etimológico del término, no vienen dados, sino que son construcciones complejas conformadas por una serie de elementos invariantes que definen su estructura interna, tal como lo plantea Samaja (1999) al presentar el concepto de matriz de datos. Samaja revisa la tesis del sociólogo noruego Johan Galtung que restringía la estructura del dato a sólo tres componentes: las unidades de análisis, las variables que en ellas se registran y los valores que dichas variables toman y agrega un cuarto componente, el indicador, el que a su vez incluye dos aspectos, dimensión (qué se mide) y procedimiento (cómo se mide) (Barriga y Henríquez, 2011).

A la tarea de delimitar el asunto más o menos concreto de la investigación se la suele denominar construcción del objeto de estudio. Ello implica que, contrariamente a lo que suele pensarse, en todo proceso de investigación, el objeto no viene dado de manera inmediata, sino que es construido por las decisiones del sujeto que investiga. Dichas decisiones se cimentan en alguna teoría en la que ese objeto se inscribe, teoría que un lector avezado será capaz de identificar, independientemente de que la misma sea explicitada o no por el investigador e incluso que este sea consciente o no de los fundamentos de dicha decisión. No en pocas ocasiones, el trabajo se lleva a cabo en el marco de un paradigma que es incorporado acríticamente y los objetos se construyen a semejanza de cómo han sido construidos por otros autores, tal como lo resumen Robert y Santoro (2002). En el marco del método hipotético-deductivo vigente en las ciencias fácticas naturales, un tema desarrollado en todo manual de metodología (Sabino, 1996; Hernández Sampieri et al., 2006; Yuni y Urbano, 2006) la delimitación del asunto de la investigación, la construcción del objeto particular de estudio se inicia con la idea disparadora del proceso. Esa idea, lejos de ser una observación ingenua, proviene de la teoría que le da fundamento, lleva inicialmente al planteo de un interrogante conceptual o problema de investigación, se continúa luego con el enunciado de una respuesta tentativa a modo de conjetura o hipótesis y termina de delimitarse en el momento en que esas definiciones iniciales se traducen al lenguaje de datos (Di Masso et al., 2014).

Los datos son, por lo tanto, una construcción que realiza el investigador a partir de un conjunto de procedimientos generativos que están muy lejos de ser meros actos de copiado de los hechos de la realidad o de registro acrítico de aquello que se le presenta al investigador a través de los sentidos. Se trata más bien de un proceso de traducciones sucesivas de los modelos teóricos a su disposición en términos cada vez más empíricos (Yuni y Urbano, 2006).

La estrategia pedagógica utilizada para trabajar el tema en el aula durante el dictado de la asignatura 'Metodología de la Investigación' en la carrera de Medicina Veterinaria de la Universidad Nacional de Rosario pone énfasis en el formato cuatripartito propuesto por Samaja y que es susceptible de ser reconocido en todo dato. Dicho formato está conformado por una entidad, una propiedad o aspecto, un estado y un procedimiento. La entidad es el objeto cognoscible acerca del cual se habla, también conocido como unidad de análisis. La propiedad o aspecto de esa unidad de análisis es la variable. El estado particular que presenta esa propiedad en el momento de observarla es su valor. Por último, el procedimiento por el cual se determina el estado que le corresponde a esa propiedad de la entidad en el momento en que se la evalúa incluye aquello que se mide o dimensión del indicador y cómo se lo mide o procedimiento del indicador. El tema se desarrolla en clase sobre la base de un texto, de lectura previa sugerida, específicamente diseñado con esa finalidad que presenta los fundamentos teóricos. Los contenidos se aplican al análisis y discusión grupal de casos concretos de resúmenes presentados en jornadas vinculadas con la investigación en ciencias veterinarias. Los ejercicios resueltos en clase al igual que en las evaluaciones parciales presentan todos la misma estructura: un enunciado que cambia y una consigna y un cuadro a completar con las respuestas por parte del estudiante que se mantienen siempre igual. Planteado de este modo, se interpreta que lo que cambia de una investigación a otra son los contenidos que conforman los datos y no la estructura que los organiza. Hablando analógicamente, aquello que llena los cuatro cajones del mueble y no los cajones en sí. Es en este sentido que, entidad, aspecto, estado y procedimiento son considerados componentes invariantes de todo dato científico. El objetivo de este trabajo fue presentar el caso de un ejercicio sencillo diseñado para evaluar el tema "componentes invariantes del dato científico" impartido como parte del programa oficial de la asignatura Metodología de la Investigación, asignatura del primer cuatrimestre del primer año de la carrera de Veterinaria de la Facultad de Ciencias Veterinarias de la Universidad Nacional de Rosario, y describir e interpretar los resultados de la estrategia didáctica empleada a partir de los resultados de dicha evaluación como un aspecto más de reflexión sobre la propia práctica docente. 


\section{Materiales y métodos}

El ejercicio en cuestión incluyó tres componentes: un enunciado representado por un breve texto que incluye los elementos vinculados con cada uno de los componentes invariantes del dato científico, una consigna de trabajo y una tabla para completar por parte del estudiante como respuesta a la consigna. En este caso particular se trabajó con el enunciado, la consigna y la tabla que se presentan a continuación:

Analiza la expresión "Los análisis de laboratorio indican que la concentración de hemoglobina en la muestra de sangre es normal" e identifica en la misma los componentes invariantes de todo dato científico.

\begin{tabular}{|l|l|}
\hline $\begin{array}{l}\text { Componente } \\
\text { invariante del dato }\end{array}$ & $\begin{array}{l}\text { Reconocimiento del } \\
\text { componente en el texto }\end{array}$ \\
\hline & \\
\hline & \\
\hline
\end{tabular}

Se analizaron 57 respuestas correspondientes al ejercicio incluido en los exámenes escritos finales de la asignatura correspondientes a los turnos de julio agosto del año 2019. En todos los casos se preservó la identidad del alumno accediendo sólo a la información del ejercicio en cuestión.

\section{Resultados}

El 38,6\% (22/57) de los estudiantes respondió correctamente a lo solicitado. El $26,3 \%(15 / 57)$ reconoció la entidad (muestra de sangre). De ellos el 13,3 \% (2/15) también reconoció la propiedad o aspecto (concentración de hemoglobina) y su valor (normal) pero no el procedimiento (análisis de laboratorio) y el $86,7 \%$ $(13 / 15)$ identificó el procedimiento y el estado, pero no la propiedad. En el 35,1\% (20/57) de los casos correspondientes a estudiantes que no identificaron correctamente a la muestra de sangre como entidad, el $35,0 \%(7 / 20)$ identificó la propiedad y el $65,0 \%(13 / 20)$, sin reconocer ni la entidad ni la propiedad, indicó correctamente el estado y el procedimiento.

\section{Discusión}

La respuesta al ejercicio propuesto para la evaluación de la internalización por parte del estudiante del concepto de dato científico, incluye varios aspectos. En primer lugar, el estudiante debe recordar los fundamentos teóricos de aquello que se le solicita en tanto la grilla a completar no incluye la denominación de cada uno de los componentes solicitados. Esos componentes no sólo tienen una denominación concreta, sino que, además, su presentación debe hacerse respetando una secuencia lógica. A continuación, debe reconocer dichos componentes en un enunciado específico. El texto que se utilizó en el caso que se presenta como ejemplo se caracteriza por exponer en forma clara y directa todos los elementos necesarios para completar la segunda parte del ejercicio (aspecto práctico) una vez que el estudiante ha completado la primera (aspecto teórico). La importancia de esta aclaración proviene de que no siempre los componentes del dato se presentan explícitamente en un texto. En ocasiones alguno de ellos está tácito como suele ser el caso del procedimiento asociado a mediciones rutinarias como el registro del peso corporal que no siempre va acompañado de la especificación de que se utilizó una balanza. Aproximadamente el $39 \%$ de los estudiantes evaluados cumplimentó satisfactoriamente ambas exigencias: el fundamento teórico (nominó correctamente y en orden lógico los cuatro componentes) y el reconocimiento práctico (identificó en el texto cada uno de dichos componentes). En aquellos que se mostraron incapaces de resolver lo planteado se pudieron identificar claramente una serie de inconsistencias llamativas. Sólo una parte de ellos reconoció a la muestra de sangre como entidad de la que se hablaba. De ellos, algunos reconocieron también a la concentración de hemoglobina como la propiedad o aspecto, es decir como la variable en estudio y el valor normal de la misma, pero no identificaron al análisis de laboratorio como el procedimiento utilizado pese a que el mismo estaba claramente explicitado en el enunciado. Este componente podría haberse soslayado en el texto en tanto podría resultar obvio que la determinación del contenido de hemoglobina en una muestra de sangre requiere de un análisis de laboratorio. Pese a que el ejemplo lo mencionaba, el procedimiento no fue reconocido. Un cierto porcentaje de los estudiantes que mencionó correctamente la entidad, identificó el procedimiento y el estado, pero no la propiedad, es decir, que manifestó que de una entidad conocida se dispone de un valor obtenido mediante un procedimiento, pero sin entender de qué variable. Este tipo de inconsistencia se hizo aún más evidente en los casos que sin poder identificar correctamente a la muestra de sangre como unidad de análisis o entidad pudieron identificar la propiedad a la que se hace referencia, como si la concentración de hemoglobina se midiera sobre la nada o bien en aquellos que, sin reconocer ni la entidad ni la propiedad indicaron correctamente el estado (valor de una variable no identificada) y el procedimiento por el cual se determinó dicho valor (algo desconocido de valor normal medido mediante un análisis de laboratorio sobre algo también desconocido). Las incongruencias mencionadas ponen de manifiesto la atomización del proceso de razonamiento frente al desafío del problema a abordar cuyo enunciado no es visto como un todo con su coherencia interna sino como una sumatoria de partes que pueden ser desagregadas como si se tratase de entidades independientes y conservar, aun así, significado. Sobre la base de la categorización de los conocimientos propuesta por Perkins (1997) puede interpretarse, en 
primer lugar, que hay un conocimiento olvidado que "ha desaparecido de la mente de los alumnos que alguna vez lo tuvieron y podrían haberlo recordado" en tanto se trataba de estudiantes que habían regularizado la asignatura, participado de la resolución y discusión de ejercicios similares que solo diferían en el texto a analizar y aprobado la evaluación parcial que incluía un ejercicio similar al del ejemplo. Sin embargo, tal como lo expresa Perkins, si bien la ignorancia relevada en este caso en particular incluye al olvido también lo excede y expone una categoría más abarcativa, la del conocimiento frágil. En tal sentido, los resultados ponen en evidencia un conocimiento sin comprensión que se manifiesta en acciones carentes de sentido y, por ende, sin significado, propio de una modalidad de aprendizaje ritual, aquel que solo sirve para cumplir con las tareas escolares. Esta vinculación con el conocimiento por parte de estudiantes universitarios es coincidente con lo observado en relevamientos previos efectuados sobre la temática (Dottavio et al., 2006, 2011; Rozados et al., 2008, 2011) y resulta trascendente como instrumento de análisis del curriculum universitario (CONEAU).

Reconocer estas modalidades que adopta el conocimiento debe conducir necesariamente a los docentes a reflexionar sobre su propia práctica, a rever cómo se enseña y cómo se aprende. Si la enseñanza y el aprendizaje se entienden como una rutina, ello lleva a presentar información que el estudiante acumula $y$ memoriza en una actitud mecánica. El resultado de esta dinámica es un conocimiento frágil y un pensamiento pobre, un conocimiento sin comprensión, con escasas posibilidades de transferencia a nuevos y diferentes contextos de aprendizaje, un conocimiento inerte que como tal se desvanece y no puede ser recuperado. Los docentes, al reflexionar sobre este trabajo son interpelados sobre la necesidad de repensar las estrategias didácticas utilizadas y probar otras formas de enseñanza que faciliten los aprendizajes que proponen desde la asignatura Metodología de la Investigación en el contexto de la carrera de Medicina Veterinaria de la UNR.

\section{Bibliografía}

Barriga OA, Henríquez GH. 2011. La relación Unidad de Análisis-Unidad de Observación Unidad de Información: Una ampliación de la noción de la Matriz de Datos propuesta por Samaja. Revista Latinoamericana de Metodología de la Investigación Social 1: 61-69.

CONEAU. La evaluación de los aprendizajes de los alumnos como instrumento de análisis del curriculum universitario. Disponible en: http://www.coneau.gob.ar/archivos/1230.pdf

Di Masso RJ, Gayol M del C, Tarrés MC. 2014. El proceso de investigación. De la idea disparadora al artículo científico. Rev. Vet. (UNNE) 25: 146-153.

Dottavio AM, Rozados VR, Di Masso RJ. 2006. La recuperación de saberes previos en los alumnos universitarios: recuerdo y transferencia. Actas VII Jornadas de Divulgación Técnico- científicas. Facultad de Ciencias Veterinarias. Universidad Nacional de Rosario. Disponible en: https://fveter.unr.edu.ar/jornadas/

Dottavio AM, Álvarez M, Canet ZE, Romera BM, Rozados VR, Di Masso RJ. 2011. El olvido inmediato: un indicador de la fragilidad del aprendizaje. Actas XII Jornadas de Divulgación Técnico- Científicas Facultad de Ciencias Veterinarias. Universidad Nacional de Rosario. Disponible en: https://fveter.unr.edu.ar/jornadas/

Hernández Sampieri R, Fernández Collado C y Baptista Lucio P. 2006. Metodología de la investigación. 4a Edición. McGraw Hill, México. 656 pp.

Perkins D. 1997. La Escuela Inteligente. Del adiestramiento de la memoria a la educación de la mente. Ed. Gedisa, Barcelona. $262 \mathrm{pp}$.

Robert MC y Santoro V. 2002. Momento metodológico de la construcción del dato. La trama de la comunicación 7: 323328.

Rozados VR, Dottavio AM, Di Masso RJ. 2008. Conocimiento frágil y olvido en el aprendizaje de la genética. Basic \& Appl. Genet. 19 (Suppl.): 216.

Rozados VR, Álvarez M, Canet ZE, Di Masso RJ, Dottavio AM. 2011. Propuesta de evaluación integral en la cursada de Genética en Medicina Veterinaria. Basic \& Appl. Genet. 41 (Suppl.): 249

Sabino CA. 1996. El proceso de investigación. Ed. Lumen Humanitas. Buenos Aires. 216 pp.

Samaja J. 1999. Epistemología y metodología. Elementos para una teoría de la investigación científica. Eudeba. Buenos Aires. $414 \mathrm{pp}$.

Taborda Chaurra J. 2010. Relación de formación y el mecanismo piagetiano de toma de conciencia. Revista Latinoamericana de Estudios Educativos (Colombia) 6: 109128.

Yuni J, Urbano C. 2006. Técnicas para Investigar 3. Análisis de datos y redacción científica. Editorial Brujas, Córdoba. 171 pp. 